This is an Accepted Manuscript of an article published by Journal of Cultural Economy: Folayan, M.

O. \& Allman, D. (2011). Clinical Trials As An Industry And An Employer Of Labour, Journal of

Cultural Economy, 4:1, 97-104, available online: https://doi.org/10.1080/17530350.2011.535376.

\title{
Clinical Trials as an Industry and an Employer of Labour
}

Morenike Oluwatoyin Folayan and Dan Allman

\section{Introduction}

To produce drugs, clinical trials depend upon the labour of volunteer participants. For ethical reasons, participants are described as volunteers because to position them otherwise could be considered undue inducement. By using a labour market perspective, we argue that clinical trial participants may be understood as workers. Ethical guidelines argue that remuneration for 'work done' by participants in research may result in coercion: research participants will feel obliged to remain in a research trial despite any discomfort and distress simply because of remunerations received. However, we suggest that monetary benefits - in the form of wages - for research participants are no more coercive than the existing and accepted level of inducement.

In our commentary, we review clinical trial conduct as it has evolved over the past two decades, the changing investments in this multi-million dollar industry, the ways in which beneficiaries and benefits are conceptualized and how these framings fall short. While it is acknowledged that framing research participants as labourers through the payment of wages has the potential to entrench inequities, we argue that it opens space to consider ways to link global health to the economic empowerment of individuals. Recognizing volunteers not as participants but as 'workers' has the capacity to widen the discourses on bioethics as a relevant and powerful counterweight to the injustices of the world today.

\section{The Clinical Trials Industry - Labourers and Volunteers}

The clinical trial industry is a provider of employment, in terms of supportive personnel, laboratory, fieldwork, pharmacy, and administrative staff. But each clinical trial involves a much larger group of people, who work on a volunteer basis. We are referring, of course, to research participants who provide vital services to this industry yet, are denied a wage and excluded from the contractual rights employees can expect to receive.

Ironically, it is bioethical guidelines that define participants' services as voluntary; the concern is that payment serves as undue inducement to research participants and undermines the quality of consent (Scocozza 1989). However, the individuals who occupy these roles may have different views of the value of their contribution to the industry. They may understand their involvement in research as services rendered in exchange for reward such as transport reimbursement, and compensation for time spent in the trial.

In developing countries, these benefits are recognized as critical components of research; because without the compensation of benefits, it would be difficult if not impossible for 
researchers to recruit and retain participants. Yet these compensations sit awkwardly with the principles of research ethics which try to balance harms against both benefit and coercion.

Benefits, although necessary and arguably, humane, can be coercive like wages as both involve the accumulation of commodities. Ethical traditions may seem to render the compensation of benefits as somehow less coercive, but would this truly be the case? Would any form of remuneration for participant involvement in a clinical trial be potentially coercive? Would this vary by the nature of the benefit, the reasons why participants may decide to enrol in a trial, and why they may choose not to continue their involvement even when the trial causes distress and discomfort? Is compensation of benefit thought off as 'less coercive' than wages because underlying it are ethical traditions that have long become instituted as norms? We argue that these benefits are not enough; that the industrial dichotomy of benefits and wages between a trials' wage-compensated labour force and its benefits-compensated participant labour force inherently is inequitable. Were trial participants to be paid wages (for daily services rendered to the trial), the coercion, we suggest, would substantially be the same.

\section{The Human Investment in Clinical Trials}

The design of clinical trials has grown more complex and more demanding for all actors and players in the field. The amount of human labour that goes into the clinical trial process has equally grown exponentially in response to the characteristics of ensuring rigorous testing of hypotheses in these ever more complex settings (Petryna 2007). Large-scale multi-country phase III trials of products of public health importance are one clear example. They frequently require thousands of research participants, and hundreds of research staff who work as administrators, outreach workers, clinicians, statisticians, engaged members of the community, and trial monitors on a truly corporate industrial scale. It is likely that the sheer breadth of human labour investment in this enterprise - especially the number of trial participants engaged - will increase as new health and disease prevention tools are sought, the ability to conduct placebo trials is reduced, and the need to rule out genetic variability in drug response becomes better understood and of greater importance (Micheal 2010).

Evolution in ways and norms of practices within the clinical trial field is not unusual. Consider the role community activists played in changing the paradigm of drug regulations to ensure prompt access to antiretrovirals. This involvement seemingly set the pace for advocacy and activism to change many conventional norms in the field of drug research and clinical trial studies. One outcome of an increased investment of public funds in clinical trials is that biomedical HIV prevention research studies themselves are increasingly being viewed as public goods. As public goods, such studies are scrutinized to ensure that they meet scientific standards in their design, are ethically conducted and address their social responsibilities. Human rights issues are more rigorously addressed and so too are the ethical and moral obligations of the research to the participants.

Benatar et al. (2005) argue that bioethical obligations to stakeholders include the need to deal with the threat of health at a global level. This entails recognition that health, human rights, and economic opportunities are all intimately linked within a complex, interdependent world. In light of this, it can be argued that there is a need to reconsider the way health and research ethics is perceived: health and research ethics are perceived: health and research ethics need to be based 
not merely on altruism, but also on a recognition and promotion of long-term self-interest. Payment for the time and services trial participants expend on clinical trials is one possible means for achieving economic redistribution as well as a means to facilitate broader access to education and basic subsistence needs in developing countries. The need for this economic redistribution can only be further justified by the economic growth witnessed in the clinical trial 'industry'.

\section{The Making of an Industry Out of Clinical Trials Research Conduct}

The current trend in the field of medical research has led to organizations being created to provide support services to the clinical trial enterprise. These include research organizations and clinical trial units that are built specifically for the conduct of clinical trials, and groups organized as research monitors and research statisticians. There are a number of such organizations in the developed world which seek to conduct clinical trials in partnership with big pharmaceuticals. There are also organizations emerging as specialists to handle the logistics needed to support extensive multi-site, multi-country research.

Funds have been committed to building the infrastructures and the human capacity required by this expanding industry, including maintenance and support between trials. This support has resulted in the pooling of substantial economic resources and investments - salaries for staff on the trial, funds for infrastructure and equipment for the institution, human capacity and prestige. There are, therefore, recognized and promoted economic benefits for actors employed by the clinical trial industry - save, of course, the trial participant.

Trial participants constitute the largest human resource within the industry and yet are not duly financially compensated for the economic value they help create for the industry. While their labour is invested in the industry, they receive the compensation only as separate from this investment - as delineated by ethics, not by economics. It might therefore be warranted to review the ethics of engagement of trial participants who function as co-labourers in the clinical trial industry, yet remain excluded by the values of volunteerism.

\section{Labourers in the Field}

Researchers have many interests and pursue these for a variety of reasons. In the cultural economies of a clinical trial's research, one interest is often the search for knowledge accompanied by 'the soft coercions' of secondary benefits such as publications, career advancement, institutional recognition, and financial support for their research. While scientific work is often experienced as more than labour, e.g. as a calling, or a benevolent contribution to the public good, the enterprise of research most definitely provides some with a source of income. In a clinical trial, employees are not framed as volunteers despite any meaning their occupation brings to their life and to the life of others. They are paid commensurately for their time and effort. Research staff expend their human resources in innovative ways to achieve the goals of research while also serving the needs of the community in which they work (Kingori 2009). The pay they receive does not appear to distract from the feeling of satisfaction and the sense of having contributed to a public good. 
In contrast, trial participants are expected to understand their role as purely altruistic, despite risks or benefits that may be associated with their 'short-term engagement' in the industry. Frequently, they receive reimbursement for their transport and some compensation for time expended. These reimbursement and compensation packages are adjusted to local wage standards otherwise it would be considered as 'undue inducement' that may lead to coercion something considered unethical. Paying research participants commensurately for the service they provide is also assumed to be unethical. This is because the compensation of benefit remuneration model is assumed to promote voluntary and altruistic participation in research, and in so doing, create a research subject free to withdraw from research participation, for example, when that participation is no longer physically and psychologically convenient or desirable. Volunteerism is assumed to enable participants to think critically about the research and participate free from consideration of personal benefit and gain (Wildman \& Hollingsworth 2009). Unfortunately, this is rarely the case. This may be due in part to the fact that underprivileged and deprived research participants within traditional cultures tend to share a nonscientific world view, and are more interested in derivable benefits from the research such as health care, monetary benefits, and less so in advancing knowledge (Benatar 2004). For many participants, clinical trials are often entangled in an individual's everyday needs and decisionmaking about health, hunger, and social capital for themselves and their families than in other more abstract notions of public good.

Further, it is assumed that voluntary trial participation means trial participants are free agents who can leave the trial at any time. Is this truly the case? In developing countries where participation in clinical trials may be a means for people to access quality care, how realistic is it that such individuals could truly discontinue participation without direct consequences? While the concept of freedom to leave a trial - a notion of volunteerism without undue reparation-is applicable in developed countries where, within-trials, standards of prevention and care may not be significantly different from that of the accessible public health service, such is seldom the case in developing countries. Indeed, one could argue that payment of wages for participating as a research subject would neither incur additional consequences nor limit the ability of research participants to discontinue participation. People also resign from regular employment if they are too distressed to continue.

Within the developing world context, the freedom of choice to participate is variable, as the coercion the benefits associated with participation produce may not leave room for true volunteerism. In these countries, clinical researchers, research staff, and the research participants may find the economic benefits and access to quality health care of greater immediate value than the scientific outcomes of the research which may be long way off or not forthcoming. Economic outcomes of research participation should be viewed as a legitimate benefit of research and should not be strategically ignored or made morally invisible. And so, while the clinical trial industry currently provides economic gains to 'employed' players and actors, the trial participants - who are arguably equal co-labourers in the industry - are left with only a feeling of having contributed to drug discovery (Ukpong \& de Cernival 2009). 


\section{Trial Participants' Engagement in Research: Can Current Indirect Payments be Revised to Wages?}

Indirect benefits for trial participants include differential access to better health care services and therapies. Free access to these in resource-poor countries is considered a legitimate obligation of the research. Yet the literature reflects with great frequency on how access to these services and commodities are a prime reason for many to participate in the research. Mazunda et al. (2008) describe how continued access to study products (in this instance, microbicidal gels) were important to trial participants for use as lubricants, for protection against HIV infection, and for increasing sexual pleasure. They also noted that participants had concerns about not being adequately rewarded. In a very real sense, the access to these services and commodities operate as a form of 'soft' coercion (Oyedeji et al. 2010), similar to the assumed effect of paying wages to participants engaged in clinical trials.

Visser et al. (2009) have noted the difficulties of ignoring the systemic and shared nature of many social and economic needs of research/trial participants in resource-poor settings, reflecting that these needs often have to be addressed through expenditures not budgeted for at the planning stage of the trial. Were trial participants to be adequately rewarded monetarily for their investment in research, the obligation and pressure to address social and economic needs could become less.

There is currently no defined procedure for addressing when, why, and how payment ought to be offered to research participants both in developed and developing countries. Grady et al. (2005) noted that most protocols did not provide a rationale for the amount a participant was monetarily compensated for their volunteerism. For the few protocols that did, the compensation was based either on what was known to be provided within comparable studies or in the same (multi-site) study, or on similar procedures across studies sometimes even within one site. The authors found little consistency between the procedural or time requirements of a study and the amount of money offered. However, studies which had some prospect of therapeutic benefit, or at least one invasive procedure, or which required a greater number of clinic visits, were associated with higher dollar amounts offered. The general lack of consistency around payment as it currently exists in the industry, and the need to standardized remuneration practices within the industry may be a platform for discussing participants' remuneration packages.

Grady et al. found also that the most important ethical concern with this advocacy for wages was related to a 'value-judgement'; that it is equally important to provide economic empowerment for trial participants who contribute significantly to the industry. These individuals could then use this income for whatever is of value to them, and thus, would not be limited to the benefits as prescribed by the research (Leach et al. 2006). While it may not be the absolute role of the trial to provide opportunities for labour, it is the responsibility of the industry and its monitors to acknowledge and recognize that addressing economic needs through payment for research participant services is legitimate and equally as acceptable as altruism as a reason for participating in research.

What are the possibilities that the payment of wages to trial participants would increase risk-taking on the part of potential trial participants? Bernstein (2003) argues that money for 
research participation could alter an individual's ability to clearly understand risks and benefits. However, in developing countries, a wage-labour model may hold no more potential for harm than current incentives, the value of which often exceed the daily income of many participants.

Applebaum et al. (1987) noted that offering payment to patient-subjects does not obviously increase their vulnerability, may reduce the financial burden of research on them, and could possibly even facilitate their understanding of research as distinct from clinical care thus minimizing any possible therapeutic misconception. Indeed, one could argue that payment of wages for participating as a research subject would not limit the ability of research participants to discontinue participation. This limitation could be no more than what currently exists within developing world contexts, where the coercive benefits associated with clinical trial participation may not leave room for true volunteerism.

Would the payment of wages for trial participation exonerate research sponsors and their institutions from any potential liabilities? This is unlikely. If clinical trials were to evolve outside an ethical context of harm, benefit and inducement, and towards one of services rendered within a labour sphere, then perhaps the industry would be better placed to re-consider the definition of potential harm. Were participants in clinical trials to transition from being remunerated participants to a form of wage labour, the industry could then review potential harm and protection of trial participants, not only from an ethical perspective, but importantly, from an occupational health and safety perspective also (see Melinda Cooper, this issue). Efforts could then be focused on health and workplace risk-reduction programmes for trial participants by the organizations conducting these clinical trials. In such a context it would not be only the ethics committees that would review protocols to ensure risk minimization and benefit maximization, social justice and fair distribution for individuals and communities engaged in the trials, but also government and industrial systems and structures would equally be in place to ensure the industry institute harm reduction measures for these 'temporary staff'- the trial participants. Within this framework, the ethical responsibilities of the research team to trial participants would be addressed as well as the ethical responsibilities of the industry Regardless of the type or form of remuneration or payment, participation as a research subject within a clinical trial is a form of labour. Recognizing this and duly compensating this as a form of wage labour may be an important step to healthier clinical trial contexts (Smith, 2009).

\section{Conclusion: Redefining the Clinical Trial Field as an Industry that Employs Labour}

This paper has presented ways in which clinical trial participants can be understood as workers. It has argued that in an increasingly mature industry like that of clinical trials, a new vantage on resources and other benefits associated with research participation and their relation to coercion warrants further consideration. As we have argued, ascribing monetary benefit in the form of wages for research participation in the current industrial context of clinical trials within the developing world may be no more coercive than existing and accepted forms and levels of inducement within the present industry.

The investments in the pursuit of research and development of medical tools and products by the clinical trial industry continue to grow like any giant industry that has a bright future. In most industries, all elements of labour in the field are duly compensated for invested time and 
efforts. While current practices in the clinical trial field do provide benefits to trial participants, these are not considered as payment for services.

We have argued that participation in research can be viewed as labour, and suggest that any such shift in remuneration could be a panacea for a range of existing ethical dilemmas in research being conducted in resource-poor settings. In so doing, we have simply sought to contribute to a young discourse which seeks to challenge traditional bioethics of clinical trial research participation with new understandings of cultural economies in order to assist the clinical trial industry to further address the situation rather than be silent on it.

\section{Acknowledgements}

The New HIV and Microbicide Advocacy Society (NHVMAS) that has continued to support engagement in national discussions around research ethics in Nigeria. The HIV Social, Behavioural, and Epidemiological Studies Unit funded by the AIDS Bureau, Ontario Ministry of Health and Long-Term Care, and the Faculty of Medicine, University of Toronto. The thoughtful critical review of this manuscript and contributions made by Ann Kelly.

\section{References:}

APPLEBAUM, P., ROTH, L., LIDZ, C., BENSON, P. \& WINSLADE, W. (1987) 'False hopes and best data: consent to research and the therapeutic misconception', Hastings Center Report, vol. 17, no. 2, pp. 20-24.

BENATAR, S. R. (2004) 'Towards progress in resolving dilemmas in international research ethics', Journal of Law Med Ethics, vol. 32, no. 4, pp. 574-582.

BENATAR, S. R., DAAR, A. S. \& SINGER, P. A. (2005) 'Global health challenges: the need for an expanded discourse on bioethics', PLoS Medicine, vol. 2, no. 7, pp. 587-589.

BERNSTEIN, M. (2003) 'Payment of research subjects involved in clinical trials is unethical', Journal of Neuro-Oncology, vol. 63, no. 3, pp. 223-224.

GRADY, C., DICKERT, N., JAWETZ, T., GENSLER, G. \& EMANUEL, E. (2005) 'An analysis of U.S. practices of paying research participants', Contemporary Clinical Trials, vol. 26, no. 3, pp. 365-375.

KINGORI, P. (2009) 'What constitutes a "good' public health researcher in the conduct of fieldwork in resource-constrained settings?', paper presented at the Publics of Public Health Conference: On Politics, Ethos, and Economy of 21st Century African Bioscience', Kilifi, Kenya, 711 December 2009.

LEACH, M., FAIRHEAD, J. \& SMALL, M. (2006) 'Where techno-science meets poverty: medical research and the economy of blood in the Gambia', West Africa. Social Science and Medicine, vol. 63, no. 4, pp. 1109-1120.

MAZUNDA, C., MUNDIA, K. \& COMMUNITY MOBILSATION TEAM (2008) 'Concerns on end of study a case for women completing 52 weeks in the MDP 301 study in Mazabuka', paper presented at the XVII International AIDS Conference: Mexico City, Mexico 38 August 2008. Abstract MOPE0852.

MICHEAL, N. L. (2010) 'Preventive HIV vaccines. Lessons from the RV 144 Thai trial', paper presented at the 5th National Conference on HIV/AIDS: The Nigeria HIV Response ownership and sustainability. Abuja, Nigeria, 3-5 May 2010. 
OYEDEJI, K., UKPONG, M. \& EZECHI, O. (2010) 'Addressing coercion in the conduct of clinical trials in microbicides', paper presented at the 2010 International Microbicides Conference: Building Bridges in HIV Prevention, Pittsburgh, PA, 22-25 May 2010. Abstract 374.

PETRYNA, A. (2007) 'Clinical trial offshored: a private sector science and public health', BioSocieties, vol. 2, no. 1, pp. 21-40.

SCOCOZZA, L. (1989) 'Ethics and medical science. On voluntary participation in biomedical experimentation', Acta Sociologica, vol. 32, no. 3, pp. 283-293.

SMITH, S. L. (2009) 'Emotional labor and the pursuit of happiness', in Unhealthy Work: Causes, Consequences, Cures, eds P. L. Schnall, M. Dobson \& E. Rosskam, Baywood Publishing Company, Amityville, NY, pp. 299-312.

UKPONG, M. O. \& DE CERNIVAL, M. (2009) 'Rethinking the value of clinical research for African Countries', 16th Canadian Conference on International Health, Ottawa, October 25-28, [Online] Available at:

http://ccih09.pbworks.com/f/a09Ukpong\%2CM.O.\%282\%29.pdf (accessed 14 November 2010).

VISSER, M., MAKIN, J. \& FORSYTH, B. (2009) 'Ethical issues in HIV/AIDS research in Africa: boundaries between research and intervention', paper presented at 9th International AIDS Impact Conference, Gaborone, Botswana, 22-25 September 2009.

WILDMAN, J. \& HOLLINGSWORTH, B. (2009) 'Blood donation and the nature of altruism', Journal of Health Economics, vol. 28, no. 2, pp. 492-503. 